\title{
Systemic Change: TEAM-UP and Beyond
}

\author{
Edmund Bertschinger \\ Department of Physics, MIT McNair 37-627, 77 Massachusetts Ave, Cambridge, MA, USA 02139
}

The AIP TEAM-UP report identifies the structural and systemic causes explaining why African Americans have not experienced the growth in bachelor's degrees in physics the way they have in other fields or as other minority groups have experienced in physics over the past two decades. Solving these problems requires changing not only the way physicists train students, but how they think about training students. Research on change in higher education suggests the need for physicists and astronomers to recognize and question the norms, values, and culture of their fields. The TEAM-UP report, SEA Change, and the new APS-IDEA all seek to stimulate and sustain a cultural transformation in the profession. 


\section{INTRODUCTION}

The race and ethnicity of US degree recipients has been reported by field of degree since 1995 [1]. Since then, the numbers of physics bachelor's degrees has more than doubled for all reported racial and ethnic groups except Black or African Americans and American Indian and Alaska Natives. Additionally, most fields in the physical sciences and engineering showed greater percentage increases in bachelor's degrees awarded to Black or African American students than physics has. Figures 1 and 2 show the comparisons.

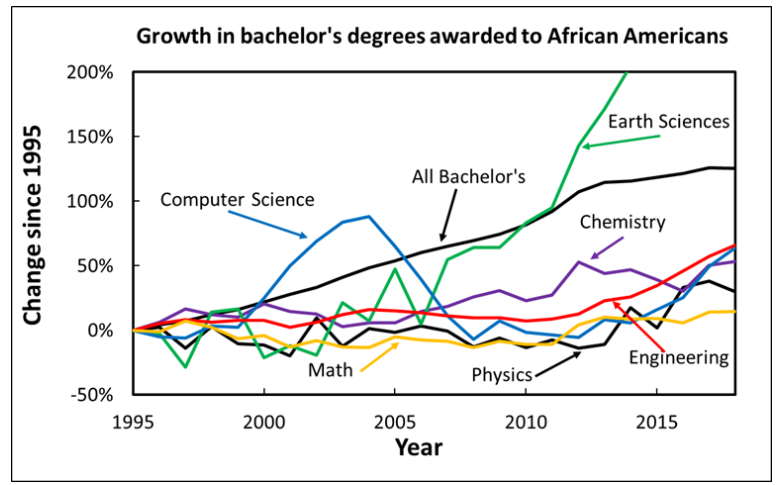

FIG. 1. Percentage change in the numbers of bachelor's degrees awarded nationally since 1995 to Black or African American students, by selected field. Astronomy is not shown because the numbers are too small over much of the time period shown. Data from NCES/IPEDS.

The comparison raises two research questions: Why does physics lag in the growth of bachelor's degrees to African Americans? What can be done about it?

In 2018, the American Institute of Physics (AIP) established a National Task Force to Elevate African American Representation in Undergraduate Physics \& Astronomy (TEAM-UP) to address these questions. After a two-year study, TEAM-UP concluded [2]:

1. "African American undergraduates have the same drive, motivation, intellect, and capability to obtain physics and astronomy degrees as students of other races and ethnicities. Many African Americans who might otherwise pursue these fields are choosing majors that are perceived as being more supportive and/or rewarding, resulting in a loss of talent to physics and astronomy."

2. The degree gap is due to the lack of a supportive environment for African American students in many departments, and to the enormous financial challenges facing them. The financial challenges affect not only individual students, but also the programs that have consistently demonstrated the best practices in supporting their success.

3. Solving these problems requires cultural shifts in how physicists educate students. The most successful departments point the way toward the necessary changes.

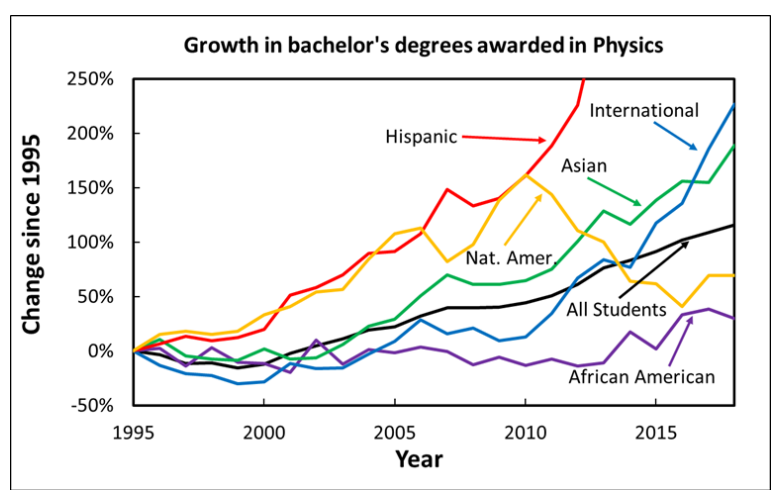

FIG. 2. Percentage change in the numbers of bachelor's degrees awarded in physics nationally since 1995 to people in different racial and ethnic groups and to international students. Students who declared two or more races or have unknown race/ethnicity are not included. Native American numbers have been smoothed with a three-year running average. Data from NCES/IPEDS.

This paper summarizes the methodology, findings, and recommendations of the TEAM-UP report. It then expands on the "What can be done about it?" question by summarizing relevant scholarship on theories of change in higher education and referencing other projects aiming to make systemic changes in physics and related professions.

Although the current research focuses on African American students in physics, Fig. 2 shows that Native Americans (American Indians and Alaska Natives) have also not fully participated in the increase in bachelor's degrees. In fact, Native Americans are the only group for which total bachelor's degrees in all fields have declined since 2010. Similarly, Fig. 1 shows that mathematics also lags in the production of bachelor's degrees to African Americans. Both of these phenomena are important topics for future research.

\section{TEAM-UP METHODOLOGY, FINDINGS, AND RECOMMENDATIONS}

The TEAM-UP project [2] conducted a mixed methods research study utilizing a strengths-based approach. Because the motivation was ultimately to help the physics and astronomy disciplines improve long-standing problems of underrepresentation, the study was designed to learn how students succeed, not how they fail. The scope of academic success was defined by the academic major requiring that the study consider the experience of undergraduate students in the corresponding department. Thus the study was designed to examine faculty interactions, department climate, and student experiences, in a manner that amplifies student voices. This section summarizes major elements of the report.

The "Why does physics lag?" research question was refined into the following set:

1. What factors lead to the persistence of African American students obtaining bachelor's degrees in physics or 
astronomy?

2. How does physics culture enhance or diminish African American students' success?

3. What are the influencing experiences?

4. What impedes or promotes culture change in the physics and astronomy community necessary for African American student persistence?

To answer these questions, TEAM-UP conducted a study that included student surveys, department chair surveys, qualitative interviews of African American students, and site visits to five exemplary physics departments. The project began by assembling bachelor's degree data from IPEDS and from the AIP Statistical Research Center to identify both long-term trends and high-performing departments (both predominantly white institutions and predominantly Black ones) that graduated many more African Americans with bachelor's degrees in physics than the average department of the same size.

The survey of department chairs (with 40 complete responses) was used to identify a subset of the high-performing departments that reported promising practices intended to recruit and/or retain African American students. Five of those departments were visited during which students, faculty, staff, and college administrators were interviewed [2].

The TEAM-UP study was similar in its use of site visits to the SPIN-UP project [3] conducted almost twenty years earlier. That project was also motivated by a decline in physics bachelor's degrees, albeit of all students, irrespective of race. By examining successful departments, the SPIN-UP authors arrived at a set of recommendations that helped improve the student experience and may have accelerated the increase in bachelor's degrees after 2005. The number of physics bachelor's degrees awarded in 2017, divided by the number awarded in 1999, is 2.50. For all STEM fields as a whole, the same ratio is 1.98 . Some of the additional growth in physics compared with other disciplines may have been a result of the SPIN-UP report. That growth did not, however, include African American students. Thus, the TEAM-UP site visits were restricted to departments that excelled in graduating African Americans in physics.

The heart of the TEAM-UP study was a qualitative interview project designed and led by researchers at the University of Maryland, College Park to assess students' planned behaviors and intentions as well as their experiences in the major [2]. TEAM-UP conducted both a survey of more than 200 physics majors (about half identifying as African American or biracial including Black or African American as one race) with snowball sampling as well as interviews of 25 black students conducted at the 2018 annual conference of the National Society of Black Physicists. The survey was designed to examine differences across race/ethnicity and gender in student motivation to pursue STEM degrees. Factors included in the design included intentions to persist, financial concerns, faculty interactions, self-efficacy, belonging, prosocial behaviors, and more. The constant comparative method was used to identify key themes and findings.

A set of common themes emerged from the site visits, surveys, and interviews, as well as existing literature. The TEAM-UP report summarized them in five factors that contribute substantially to the success of African American students majoring in physics:

1. Belonging

2. Physics identity

3. Academic support

4. Personal support

5. Leadership and structures

Brief examples of the research supporting these themes follow.

A sense of belonging has long been recognized as a fundamental mediator in learning [4]. Its importance is often clearest in its absence. For example, one survey respondent wrote,

The climate of the physics department is very non-inclusive of people of color.... They would say [things] like "You should change your major."

Physics identity - the perception that one is, or is preparing to become, a physicist-is another crucial factor in persistence in physics (or other STEM disciplines) [5-8]. It, too, is most often recognized through impediments. This example comes from an inteview,

I've had two professors ask me why I'm in physics... "Why are you still a physics major? ... "You're making your life difficult doing all this." It's very discouraging when you hear [this].

Academic support includes effective teaching and mentoring using strength-based approaches. The faculty role is paramount and includes the development of personal bonds [9]. A good example is this quote from a student,

There was one teacher that-really, honestly, I was going to give up on physics and she changed everything....

Personal support is necessary not only to navigate the classroom and campus, but even to be present in them. Financial concerns were largest for African American students compared with other racial and ethnic group members in the survey and they were also apparent in the site visits. One student noted the challenges of obtaining adequate support to continue as a physics major:

I am paying the cost of college on my own, so that is continuous financial stress. Working 20plus hours a week, including overnights.... Lack of support from the college/department.

Financial stress is especially great for African American students because of the 1:10 ratio of accumulated wealth for Black families compared with white families in the U.S. [10].

Leadership and structures are required for physics departments and their host campuses to create conditions of belonging that enhance physics identity development and provide 
the academic and personal support necessary for students to thrive. As one faculty member noted in a site visit, "Building community takes a lot of sweat equity." In the most successful departments, all physics faculty members are invested.

The TEAM-UP report provides a detailed set of findings and recommendations for each of the five factors [2]. The overall conclusion of the report is that two broad factors explain why physics lags other fields in the production of bachelor's degrees to African Americans. The first lies within the physics community itself: many departments lack the conditions and commitment described above and found in the most successful departments. The second factor, the financial challenges many facing African American students, does not arise from within the departments. However, it affects many of the most successful departments, which often have the least resources. Examples include predominantly Black institutions and HBCUs [11, 12].

Like the SPIN-UP report [3], the TEAM-UP report provides numerous recommendations for creating a more supportive department where students thrive, most of them costing little beyond faculty and leadership time and commitment [2]. For each of the five factors, the report gives findings describing practices of the most successful departments; the corresponding recommendations suggest ways other departments can implement these practices. The TEAM-UP authors recognize that achieving these outcomes will require more than a report; it will require thoughtful efforts in change management, as described next.

\section{THEORIES OF CHANGE}

Whenever an individual, organization, institution, or social movement seeks intentionally to change something, a theory of change is used, even if that fact is not recognized. At its simplest, a theory of change is a description of how and why change is expected to happen in a particular context [13].

In organizational theory, it is helpful to distinguish two different kinds of change [14]. First-order change deals with conditions were there exists agreement about the desired outcome but there may be disagreement about the optimal method. Often the change involves improvements or adjustments to an existing system. Second-order change deals with conditions where there is no agreement about the existence and nature of the problem to be solved, or the problem is not viewed as requiring solution. In these cases, the system itself may be part of the problem. The underlying norms, values, and culture of the organization, institution, or society must be addressed before the desired change can occur.

When a problem requiring second-order change is addressed using first-order methods, the result is most often failure. For example, in the context of pre-college education, Sarason [15] wrote, "[I]f you want to change and improve the climate and outcomes of schooling both for students and teachers, there are features of the school culture that have to be changed, and if they are not changed, your well-intentioned efforts will be defeated."

The TEAM-UP authors conclude that changing the outcomes for African American physics students requires second-order change, and therefore success requires the physics community to grapple with its own norms, values, and culture. This is particularly challenging for a profession that has been described as having a culture of no culture [16].

To its five factors characterizing successful departments in the preceding section, the TEAM-UP report adds a sixth category of recommendations on change management [2]. These recommendations involve understanding and developing theories of change that apply not only to a particular department, but also to the college or university within which that department exists, to the physics profession at large, and to the individual physicists and astronomers who may not agree about the existence or nature of a problem. Professional societies, as bodies that both represent physicists and establish norms, play an important role in the TEAM-UP report's theories of change.

TABLE I. Example of a Change Framework.

\begin{tabular}{l}
\hline \hline Type of Change \\
\hline Content of change: TEAM-UP Belonging recommendations \\
Degree of change: second order \\
Levels of change: group (faculty, students) \\
Focus of change: structures, behaviors, attitudes \\
Source of change: internal, professional societies \\
\hline Context for Change \\
\hline Political factors: social pressures from students \\
External stakeholders: APS, AAAS \\
Institutional status: challenging organizational values \\
Institutional culture: faculty shifting attention from \\
graduate to undergraduate education, EDI \\
Capacity and readiness: Some capacity and readiness \\
\hline Agency/Leadership \\
\hline Top down vs bottom-up: dept chair has influence, some authority; \\
existing faculty relationships build agency; students \\
influence dept chair but have limited agency \\
Collective leadership: builds collective leadership among \\
the administration, faculty \\
Shared leadership: departmental action team (DAT) builds \\
shared leadership between students, staff, faculty \\
\hline Approach to Change \\
Political theory: student petitions, create coalitions, \\
faltural theory: departmental values statement; \\
\hline \hline
\end{tabular}

Second-order change is a nonlinear process involving mul- 
tiple inputs for many interacting variables. A narrative framework [14] can capture the full complexity much better than a mathematical model. Table I shows an example for a physics department wishing to implement the Belonging recommendations of the TEAM-UP report. These recommendations often require faculty to change not only how some courses are taught, but, more importantly, how some faculty view their role. This fictional department resides in a researchintensive university whose faculty prize graduate education and research above undergraduate teaching.

Table I reflects the first stage in effective planning for organizational change. This department is attempting to leverage participation in two projects organized by professional societies (APS, AAAS) focusing on equity, diversity, and inclusion (EDI) to build their capacity for cultural transformation. Such change efforts in higher education typically require more than 5 years to achieve their goals [17].

Research shows that successful change must take account of the type of change, the departmental context, the agency and leadership of stakeholders, and the detailed theories of change. In Kezar's framework [14], each topic listed under these categories in Table $\mathrm{I}$ is to be filled in by the change agents (e.g., "TEAM-UP Belonging recommendations"). Multiple theories of change are listed in the "Approach to Change" box. That is because theories of change, like models of nature, have assumptions and limitations. Political theories of change consider social power and hierarchies, believing these to be important in describing change. Cognitive theories of change consider how people learn because changing structures, behaviors, or attitudes generally requires unlearning some things before learning others. Cultural theories give weight to symbols and meanings expressed through norms and values. Completing this table, and learning more about the relevant theories of change, is a crucial step in effective change management.

These theories of change differ from the traditional strategic planning approach used by physicists, which is called scientific management in the social sciences. Scientific management, institutional, and evolutionary theories of change (the latter considering the effect of external drivers) are often efficient and effective for first-order change, but they are inadequate for second-order change.

\section{CULTURAL CHANGE PROJECTS IN PHYSICS AND RELATED FIELDS}

During the last several years, numerous efforts have begun to advance cultural change in physics and related fields. Besides TEAM-UP, these efforts include the APS Inclusion, Diversity, and Equity Alliance (APS-IDEA) [19]; the APS Effective Practices for Physics Programs (EP3) [20]; the Inclusive Graduate Education Network (IGEN) [21]; and STEP UP [22]. All of these projects are led or co-led by physi- cists or physics education researchers. In addition, there are graduate student-initiated and led projects in physics [23] and astronomy [24]. The STEMM Equity Achievement (SEA) Change project of the AAAS [18] works across disciplines including physics.

Although these efforts represent a great variety of methodologies, they share common values and vision and have some common features. First, all create a community of practice. Communities of practice reflect the social nature of learning in ways that embody EDI [25]. Most of the efforts also take advantage of networks of looser affiliations that can bring resources to community members. Communities of practice dedicated to second-order change have been dubbed communities of transformation [26].

Cultural change efforts also engage in sensemaking, a process of learning in community with others. Sensemaking is an essential precursor to change when stakeholders disagree about the existence, nature, or importance of the problem to be solved [27]. Sensemaking is fundamental to cognitive theories of change [14], which posit that people will not agree to changes they do not, or can not, understand.

A second principle underlying some, but not all, of the efforts is shared leadership. Shared leadership has been described as an improvement on the leader/follower binary that allows organizations to benefit from the leadership of many individuals [28]. It is an important ingredient of many successful reforms in higher education.

Here a modified definition is adopted. Shared leadership is the equitable collaboration of all relevant stakeholders in creating and assessing change. It is closely related to participatory democracy and to participatory action research. Shared leadership gives relatively more power to those with the most to gain from change but the least power to achieve it themselves. This idea is embodied in the first principle of Quan et al. [29]: Students are partners in the educational process.

The existence of multiple cultural change initiatives offers an excellent opportunity for research in institutional change [30]. It also presents an opportunity for efforts with similar goals to form alliances following the model of a social movement. Through shared leadership operating across all levels from individuals, through the profession, and into society at large, social movements have potential for greatly accelerating cultural change in physics and beyond [31]. Such efforts may be necessary for the widespread implementation of the TEAM-UP recommendations.

\section{ACKNOWLEDGMENTS}

This publication would not have been possible without the amazing work of my colleagues on the TEAM-UP task force [2]. I also thank Rebecca Lindell for her encouragement and guidance, and anonymous reviewers for helpful suggestions. 
[1] Integrated Postsecondary Education Data System, IPEDS Completions Brochure, National Center for Education Statistics Publication No. 2019007. https://nces.ed.gov/pubs2019/ 2019007.pdf. Retrieved 6/4/2020.

[2] B. Beckford et al., The Time is Now: Systemic Changes to Increase African Americans with Bachelor's Degrees in Physics and Astronomy (American Institute of Physics, College Park, MD, 2020). https://www.aip.org/sites/default/files/ aipcorp/files/teamup-full-report.pdf. Retrieved 6/4/2020.

[3] R.C. Hilborn, R.H. Howes, and K.S. Crane, Strategic Programs for Innovations in Undergraduate Physics: Project Report (American Association of Physics Teachers, College Park, MD, 2003). https://www.aapt.org/Programs/projects/spinup/ upload/SPIN-UP-Final-Report.pdf. Retrieved 6/4/2020.

[4] G. Trujillo and K.D. Tanner, Considering the Role of Affect in Learning: Monitoring Students' Self-Efficacy, Sense of Belonging, and Science Identity, CBE-Life Sci. Educ. 13(1), 6 (2014).

[5] H.B. Carlone and A. Johnson, Understanding the science experiences of successful women of color: Science identity as an analytic lens, J. Res. Sci. Teach. 44(8), 1187 (2007).

[6] P.R. Aschbacher, E. Li, and E.J. Roth, Is science me? High school students' identities, participation and aspirations in science, engineering, and medicine, J. Res. Sci. Teach. 47(5), 564 (2010).

[7] Z. Hazari, P.M. Sadler, and G. Sonnert, The Science Identity of College Students: Exploring the Intersection of Gender, Race, and Ethnicity, J. College Sci. Teach. 42(5), 82 (2013).

[8] S. Hyater-Adams, C. Fracchiolla, T. Williams, N. Finkelstein, and K. Hinko, Deconstructing Black physics identity: Linking individual and social constructs using the critical physics identity framework, Phys. Rev. Phys. Educ. Res. 15, 020115 (2019).

[9] S. Fries-Britt and D. White-Lewis, In Pursuit of Meaningful Relationships: How Black Males Perceive Faculty Interactions in STEM, Urban Review (2020).

[10] J. Bricker, L.J. Dettling, A. Henriques, J.W. Hsu, L. Jacobs, K.B. Moore, S. Pack, J. Sabelhaus, J. Thompson, and R.A. Windle, Changes in U.S. Family Finances from 2013 to 2016: Evidence from the Survey of Consumer Finances, Federal Reserve Bulletin 103(3), 1 (2017).

[11] Q.L. Williams, Undergraduate physics programs at HBCUs: Can we stop the losses? Phys. Today 63(6), 47 (2010).

[12] K.L. Williams and B.L. Davis, Public and Private Investments and Divestments in Historically Black Colleges and Universi- ties (American Council on Education, Washington, DC, 2019).

[13] https://www.theoryofchange.org/what-is-theory-of-change/. Retrieved 6/4/2020.

[14] A. Kezar, How Colleges Change: Understanding, Leading and Enacting Change (Routledge, New York, 2018), 2nd edition.

[15] S. Sarason, Revisiting the Culture of the School and the Problem of Change (Teachers College Press, New York, 1996), p. 340.

[16] S. Traweek, Beamtimes and Lifetimes: The World of HighEnergy Physicists (Harvard University Press, Cambridge, MA, 1988).

[17] S. Elrod and A. Kezar, Increasing Student Success in STEM: A Guide to Systemic Institutional Change (AAC\&U, Washington, DC, 2016).

[18] https://seachange.aaas.org/. Retrieved 6/4/2020.

[19] https://www.aps.org/programs/innovation/fund/idea.cfm. Retrieved 6/4/2020.

[20] http://ep3guide.org/. Retrieved 6/4/2020.

[21] https://www.apsbridgeprogram.org/igen/. Retrieved 6/4/2020.

[22] https://engage.aps.org/stepup/home. Retrieved 6/4/2020.

[23] https://accessnetwork.org/. Retrieved 6/4/2020.

[24] https://www.tamiastronomy.org/. Retrieved 6/4/2020.

[25] E. Wenger, R. McDermott, and W.M. Snyder, Cultivating Communities of Practice: A Guide to Managing Knowledge (Harvard Business Review Press, Cambridge, MA, 2002).

[26] A. Kezar, S. Gehrke, and S. Bernstein-Sierra, Communities of Transformation: Creating Changes to Deeply Entrenched Issues, J. High. Educ. 89(6), 832 (2018).

[27] A. Kezar, Understanding sensemaking/sensegiving in transformational change processes from the bottom up, High. Educ. 65, 761 (2013).

[28] A.J. Kezar and E.M. Holcombe, Shared Leadership in Higher Education: Important Lessons from Research and Practice (American Council on Education, Washington, DC, 2017).

[29] G.M. Quan et al., Designing for institutional transformation: Six principles for department-level interventions, Phys. Rev. Phys. Educ. Res. 15, 010141 (2019).

[30] K. Quardokus Fisher and C. Henderson, Department-Level Instructional Change: Comparing Prescribed versus Emergent Strategies, CBE-Life Sci. Educ. 17(4) (2018).

[31] M. Pastor and R. Ortiz, Making Change: How Social Movements Work and How to Support Them (University of Southern California, Los Angeles, 2009). https://dornsife.usc.edu/pere/ making-change/. Retrieved 6/4/2020. 